\title{
The Contingency Problem for Neo-Conventionalism
}

\section{Introduction}

Traditional conventionalism about modality claims that a proposition is necessarily true iff it is true by convention. In the wake of the widespread repudiation of truth-by-convention, traditional conventionalism has fallen out of favour. However, a family of theories of modality have arisen that, whilst abandoning truth-by-convention, retain the spirit of the traditional conventionalist theory of necessity. Call such theories 'neo-conventionalisms'. These theories surpass their forebears and do not fall victim to criticisms inherited through truth-by-convention. However, despite appearances, not all criticisms of traditional conventionalism target truth-by-convention. Any conventionalist theory of modality must face the contingency problem, which claims that the necessity of the conventional necessities is jeopardised by the contingency of our linguistic conventions.

There are several ways of characterising the problem, but the primary tension stems from the desire to maintain:

(CONTINGENCY) We could have held conventions other than those we actually do.

And the validity of the characteristic axiom of the $\mathrm{S} 4$ system: $^{1}$

(S4) if nec(P) then nec(nec(P)).

The goal of conventionalism is to present a sensible antirealist theory of modality: antirealist in that it maintains that modality is ultimately explained in terms of our conventions, and sensible in that it does not commit one to the truth of the kinds of counterfactuals that require a model that acts as a countermodel to the validity of S4. ${ }^{2}$ This can be done by showing that there is a framework for conventionalism where $\mathrm{S} 4$ is valid. Adopting such a framework would mean that the conventionalist is not committed to a countermodel to the validity of $S 4$, and thus can decide on their own terms if it is a principle they want to maintain.

Herein I argue that both the traditional conventionalist and the neo-conventionalist must address the contingency problem (despite the claims of some prominent neo-conventionalists (Cameron (2010a), Sider (2003)) that their positions are safe from it). I examine a response from Einheuser (2006) that builds upon a response from Wright (1985). I show that the Einheuser response does more to accommodate the conventionalist's modal beliefs, but that it does not fully satisfy some further conditions that ought to be laid upon such a response. I then suggest how the response can be revised so as to satisfy these conditions. The resulting conventionalist framework is compatible with the validity of $\mathrm{S4}$, and suitably in the spirit of conventionalism.

\section{Neo-Conventionalism}

When considering necessity it is worth distinguishing between what I shall call 'necessitation claims',

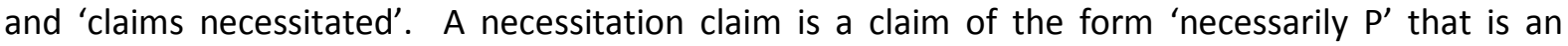
attribution of necessity. A claim necessitated is a claim ' $P$ ' to which necessity is attributed (but that need not be an attribution of necessity itself). So, for the necessitation claim 'necessarily $\mathrm{P}^{\prime}$ ', the

\footnotetext{
${ }^{1}$ That is, that the $\mathrm{S} 4$ axiom is true in every model (where a model is a complete description of the pluriverse).

${ }^{2}$ It is debatable whether the conventionalist should be required to maintain the S4 principle. Both Wright $(1980,1985)$ and Hale (2002) suggest that to insist upon S4 is to beg the question against the conventionalist. Lewy (1976) argues that the contingency of nec(P) jeopardises the necessity of $P$, as do Van Cleve (1996) and Sidelle (2009). See also Elder (2006) for a related criticism of conventionalism. Here I assume that if the conventionalist can maintain the validity of S4, then they should.
} 
claim necessitated is P. ${ }^{3}$ The traditional conventionalist can be characterised as claiming that the claims necessitated are true-by-convention and that this makes the corresponding necessitation claims true. For example, 'vixens are foxes' is true because by definition being a fox is part of what it is to be a vixen, and it is necessary because it is true-by-convention. Neo-conventionalism retreats from this position. It still attributes the necessity of the claims necessitated to convention, but rejects truth-by-convention. 'Vixens are foxes' is true because each and every vixen is a fox (say), but it is necessary because of our conventions. One might think that the neo-conventionalist is moving from truth-by-convention for the claims necessitated to truth-by-convention for the necessitation claims. This however is a conclusion that the neo-conventionalist resists. Neither 'vixens are foxes', nor 'necessarily, vixens are foxes' is true-by-convention. Neo-conventionalist explanations of necessitation claims like 'necessarily, vixens are foxes' take the form: 'necessarily, vixens are foxes' is true iff some fact $F$ holds, where what counts as an instance of $F$ is determined by convention. As such, the necessitation claim is true not in virtue of convention, but in virtue of $F$ holding. Analogously, 'Jon and Michaela are married' (M) is true because they had a wedding ceremony and we hold the convention that performing such a ceremony makes people married. $M$ is not true-by-convention, it is true because a fact $F$ (the ceremony happening) holds, and we hold the convention that $\mathrm{F}$ is sufficient for the truth of $\mathrm{M}{ }^{4}{ }^{4}$

Here I consider two forms of neo-conventionalism: Cameron's Deflationism (2009, 2010a, 2010b), and Sider's Humean account $(2003,2011){ }^{5}$ Deflationism aims to explain the truth of the necessitation claims, making the familiar equivalence that a necessitation claim nec $(P)$ is true iff the claim necessitated $\mathrm{P}$ is true in all of the possible worlds. The role of convention is to ground the difference between the possible and impossible worlds. The position starts with an actualist possible worlds account. Worlds are abstract entities (Cameron's preference is for sets of propositions). Such accounts are often faced by challenges to their reductive ambitions. They encounter a problem in explaining which worlds are possible and which are impossible. This allegedly cannot be done without taking some modal notion (often consistency) as primitive. Cameron (2009, p.13) claims that the distinction between the possible and impossible worlds is genuine and mind-independent, but highly unnatural. That is, there is a distinction, and it doesn't depend at all on what we think about it, but it was decided upon by us, and does not represent any metaphysical difference between the things distinguished (such as a natural property shared by all of one group and none of the other). To borrow the metaphor, the distinction does not carve reality at nature's joints; it is not reflected in the deep metaphysics of the world. All that distinguishes between members of one group and members of the other is that some are assigned to one group, and some to the other. The distinction is real, it's just nothing special. Frege gives similar remarks about the equator:

We often speak of the equator as an imaginary line; but it would be wrong to call it a fictitious line, it is not a creature of thought, a psychological process, but is only recognized or apprehended by thought. If to be recognized were to be created, then we should be able to say nothing positive about the equator for any period earlier than the date of its alleged creation. (Frege (1884), p.35)

\footnotetext{
${ }^{3}$ I phrase my discussion in de dicto linguistic terms for the sake of making it compatible with traditional conventionalism. This allows for bridging between traditional and neo-conventionalism, and highlights the difference in how they work. However, I trust that all that follows could be phrased in terms of non-linguistic necessities. This is compatible with neoconventionalism, and with the major discussion in the paper. I trust that nothing substantial hangs on this

${ }^{4}$ One might worry that ' $\mathrm{F}$ is sufficient for $\mathrm{M}$ ' is true-by-convention. This is true, but only in so far as it is an object-language formulation of the convention in play. This is truth-by-convention at its most innocuous - equivalent to saying that 'suchand-such actions are a wedding ceremony' is true-by-convention because convention determines what constitutes a wedding ceremony. The important point is that $\mathrm{M}$ is true not by convention, but because of $\mathrm{F}$, and that truth-byconvention doesn't feature in the explanation of necessity in any way that is traditionally found to be problematic.

${ }^{5}$ One should not confuse Cameron's position with a deflationary theory of truth; rather, it provides deflationary truth conditions for modal claims.
} 
To use a new analogy: I invent a new board game, and I'm deciding on the size of the board. There are many potential sizes I can pick: $10 \times 10,30 \times 30,30 \times 50$, etc. I pick $30 \times 30$ because of reasons relevant to the game mechanics, ergonomics, etc., but other options were equally viable (in particular, at least some were viable considering those same considerations relevant to the game mechanics). Once I make it, the board is genuinely that size. It is not mind dependent (in Cameron's sense) that the board has a $30 \times 30$ layout. However, it is conventional that the game board is that size, because I could just as easily have picked another size. This doesn't mean that there was nothing in the choice. I had good reason to pick that size; it just wasn't a reason that had anything to do with metaphysics.

Sider $(2003,2011)$ presents a similar account. Instead of claiming that nec(P) iff $P$ is true at all possible worlds, Sider says that nec $(P)$ iff $P$ is true and $P$ is a claim of a certain kind. The kinds of claim in question are selected by convention (just as the deflationist claims that the possible worlds are selected by convention). For example, if analytic, logical, and mathematical claims are the kinds selected, then nec(P) iff $\mathrm{P}$ is a true claim that is analytic, logical, or mathematical.

Convention selects a set of true sentences to act as modal axioms; then selects a set of truth preserving relations between sentences and groups of sentences to act as modal rules. For any such collection there is a corresponding set of modal theorems (the closure set of the modal axioms under the modal rules, which is the intersection of sets that contain every modal axiom, and contain every sentence to which a subset of its members bears a modal rule relation). According to the Humean, these modal theorems are the claims necessitated. This framework gives the Humean the ability to accommodate necessity in any realm of discourse. In order for the theory to account for some branch of necessity, it need only add the appropriate claims to the modal axioms.

\section{The Contingency Problem}

Both Cameron and Sider take their positions to be immune to the contingency problem. Sider (2003, p.28) says "[t]he contingency objection was that conventionalism turns statements about logic and mathematics into statements about conventions, which then inherit the contingency of conventions. The present theory has no such consequence". Cameron (2010a, p.11) characterises the contingency problem as stemming from the truth-by-convention of propositions such as $<$ necessarily, $\mathrm{P}\rangle$, and $<$ there are necessary truths $>.{ }^{6}$ As such, he claims that the neo-conventionalist needn't reject S4, because they do not rely on truth-by-convention (of either the claims necessitated, or the necessitation claims). Both identify truth-by-convention as essential to get the problem off the ground (and think themselves safe because it does not feature in their positions). However, whilst truth-by-convention is sufficient for the problem to take hold, it is not necessary. ${ }^{7}$ All that is necessary is that the necessitation claims be in some way determined by convention. For this to be the case it is sufficient that the following counterfactual be true:

(A) If our conventions had been suitably different, then a different range of necessitation claims would have been true.

We can show that $A$ is inconsistent with $\mathrm{S} 4$ with the following argument:

\footnotetext{
${ }^{6}$ This is in fact a mild mischaracterisation. Cameron gives this reasoning as motivation for the conventionalist to sit on the contingency horn of Blackburn's dilemma, according to which the source of necessary truth must itself be either necessary or contingent. If the source of necessity is itself necessary, then regress ensues; if the source of necessity is contingent, then the necessity of those necessitation claims "has not been explained or identified, so much as undermined" (Blackburn (1993), p.53). However, from Cameron's arguments I take it that he thinks these considerations are sufficient to dispel the contingency problem (so characterised).

${ }^{7}$ There are some formulations of the contingency problem for which truth-by-convention is a necessary condition. Van Cleve (1994) gives a particularly strong formulation of the problem, and a further argument for the conclusion that everything is contingent. However, Van Cleve's argument relies on truth-by-convention to apply to conventionalism. The neo-conventionalist is not threatened by the argument because they reject truth-by-convention. As such I will not consider Van Cleve's argument further here.
} 
(VS4) The axiom scheme $\operatorname{nec}(\mathrm{P}) \rightarrow \operatorname{nec}(\operatorname{nec}(\mathrm{P}))$ is valid (any proposition that is necessary is necessarily necessary).

(A) If our conventions had been suitably different, then a different range of necessitation claims would have been true.

(CONTINGENCY) We could have held conventions other than those we actually do.

(4) If a different range of necessitation claims were true, then at least one necessitation claim that is actually true would be false. ${ }^{8}$ Let $Q$ be an arbitrary claim that is necessary but would not have been necessary had our conventions been different.

(5) $\neg($ nec $(Q) \rightarrow$ nec $(\operatorname{nec}(Q)))(Q$ is necessary but it could have been that $Q$ was not necessary.) (From 4 and CONTINGENCY)

(6) The axiom scheme $n e c(P) \rightarrow \operatorname{nec}(n e c(P))$ is valid and invalid (contradiction from 5 (because 5 provides a countermodel to S4) and VS4 (which insists that there are no countermodels to S4)).

If the conventionalist maintains $A$ then they are committed to a countermodel to $S 4$, and must reject its validity. As such, there is reason for the conventionalist to reject $A$. However, there is a sense in which the conventionalist wants $A$ to be true, because without the truth of something akin to $A$, it is not clear how convention could be playing a role in determining necessity. This is in violation of LINK:

(LINK) There must be a clear link between our conventions and the true necessitation claims. ${ }^{9}$

Maintaining LINK should be considered essential to any conventionalist position. A conventionalist account that violates LINK (or an equivalent principle for conventionalisms about realms of discourse other than the modal) cannot be said to be a conventionalism at all, for the conventions cannot be said to be doing the theoretical work in determining the range of modal facts. In light of the desire to maintain S4, and the importance of LINK, the contingency problem can be characterised as the desire for both $A$ and $\neg A$ to be true. We want $A$ to be true because it gives us a link between our conventions and the true necessitation claims (satisfying LINK), and we want $\neg A$ to be true because $A$ commits the conventionalist to the invalidity of S4, as it provides a model where some claims necessitated are only contingently necessary. Wright (1985, p.190) responds to this problem by suggesting that our modalising is governed by the convention:

(RIGIDITY) What it is true to say of a hypothetical state of affairs, and what it is true to say in a hypothetical state of affairs, is to be determined by reference to our actual linguistic conventions, even if those are not the conventions that would then obtain.

Wright motivates RIGIDITY with several examples that he takes to be uncontroversial, such as "[i]f 'red' had meant what 'blue' now means, the sky would have been blue" (Wright (1985), p.190). ${ }^{10}$ RIGIDITY allows the conventionalist to maintain S4, as even when we consider worlds where the inhabitants hold different conventions, we still make modal judgements relative to our own conventions. However, this is not the whole story. If we take RIGIDITY as the entire response then we still fail to respect LINK. RIGIDITY leaves no room for covariance across worlds between conventions and the range of true necessitation claims. This means that there is no sense in which $A$ is true, because no matter what hypothetical situation we entertain, even one in which we have

\footnotetext{
${ }^{8}$ It might be that the difference alluded to in $A$ is that there is a necessitation claim $P$ that is actually false that would be true. This would render the actually true necessitation claim nec( $-\operatorname{nec}(P))$ false, so either way the result is as described in 4. This relies on $S 5$, but for those unwilling to accept $S 5, A$ could just as easily stipulate the required change.

${ }^{9}$ Exactly how LINK should be phrased may be debatable, but something like it should hold. Einheuser (2006) p.470 recognises that conventional features of reality must systematically covary with changes in our conventions. Sidelle (2009) p.231 also recognises this need, as does Elder (2006). LINK is in this spirit.

10 For further discussion motivating RIGIDITY, see Sidelle (2009).
} 
different conventions, the same range of necessitation claims will be true, rendering $A$ false. It was the truth of $A$ that was allowing us to maintain LINK (because if $A$ is true then there is at least some covariance between our conventions and which necessitation claims are true, providing a link between them), so maintaining RIGIDITY is in conflict with LINK because if RIGIDITY is true then A cannot be, and if $A$ is not true then that removes the only support we had for LINK. Without LINK it is not clear how our conventions can actually play a role in determining the necessitation claims. As such, the conventionalist must take further steps to either allow A to be true in some sense, or to secure the truth of LINK by some other means.

Wright might claim that RIGIDITY does not actively conflict with a claim of covariance between our conventions and the necessitation claims, but rather masks that covariance. ${ }^{11}$ As such RIGIDITY does not compel us to reject LINK. However, RIGIDITY does conflict with A; whilst it may not give the lie to LINK, further support for it still must be found. This is the task that I take Einheuser to embark upon.

\section{The Einheuser Framework ${ }^{12}$}

Einheuser (2006) introduces a framework by which we can provide a response in the spirit of RIGIDITY, whilst respecting LINK. She distinguishes between our conventional practices (the behavioural regularities - such as always driving on the left, or calling all female foxes vixens - that constitute our conventions) and the carvings (the theoretical counterpart to our conventional practices) that correspond to them. ${ }^{13}$ According to Einheuser, whenever we talk of conventions we might mean either conventional practices, or carvings. Exploiting this ambiguity, Einheuser introduces a possible worlds framework by which we can model conventional possibility.

There are two kinds of feature a world might have. S-features are determined by the 'preconventional' world (i.e. features that are in no way determined by convention). C-features result from the application of conventions to the world. In the case of conventionalism about modality, the $c$-features are (for the neo-conventionalist) those features that correspond to the necessitation claims (or, for the traditional conventionalist, the claims necessitated). The $s$-features are all nonmodal features of the world (assuming conventionalism only about modality).

There are possible and impossible worlds. According to Einheuser, worlds in the framework are ordered pairs of substrata and carvings $\langle s, c\rangle$ and there is a world for every substratum/carving combination. A substratum is the sum of the $s$-features in a world (one should ignore the philosophical connotations of the term 'substratum', and take it merely to be the way the world is, minus those facts that are in some way determined by convention). A carving acts as a function from a substratum to a set of $c$-features. Einheuser describes a carving as imposing structure on the substratum. ${ }^{14}$

A substratum $s$ supports a feature $F$ iff it contains all $s$-features necessary for a world containing it to have $\mathrm{F}$. Einheuser's preferred example comes from conventionalism about ontology. If mountains are conventional, then for $s$ to support mountains is (say) for it to contain sufficiently altitudinous regions. For $s$ to fail to support mountains is for it to be sufficiently 'flat'. A world $w$ supports $F$ iff its substratum supports $F$. A carving $c$ carves a $c$-feature $F$ iff it would yield $F$ in any

\footnotetext{
${ }^{11}$ He writes to this effect in (1985), p.192

12 The semantics Einheuser presents are controversial. However, Einheuser thinks it justified in responding to the contingency problem. In exploring her response I assume their acceptability.

${ }^{13}$ For the sake of simplicity, I follow Einheuser in assuming that each world contains only one set of conventional practices. Whilst this is likely false, it makes the following discussion easier, and a full discussion of the consequences of multiple sets of conventional practices is beyond the scope of this paper.

${ }^{14} \mathrm{I}$, Like Einheuser, will have little to say about what exactly a carving is beyond that it is that which our conventional practices give rise to. Einheuser describes it as a theoretical concept that serves as an abstraction from the conventional practices that constitute our conventions. As such, carvings are neither $s$-features nor $c$-features, but the bridge between the two.
} 
world that supports F. ${ }^{15}$ In effect, a carving that carves mountains makes it the case that the altitudinous region is a mountain, rather than just an area of high elevation. A world $w$ carves $F$ iff its carving carves $F$. There are three ways that a world could lack some $c$-feature. The substratum could fail to support it, the carving could fail to carve it, or both the substratum and the carving could fail.

A carving $c$ is grounded by a substratum $s$ (represented $c_{s}$ ) when $s$ supports the conventional practices (which are themselves part of the substratum) that correspond to $c$. A world $w$ grounds a carving $c$ when its substratum grounds $c$. Worlds where the substratum of the world grounds the carving of that world are called 'diagonal worlds' (represented $\left.\left\langle s, c_{s}\right\rangle\right) .{ }^{16}$

Since there are two dimensions along which modal change can occur, change in substratum, and change in carving, Einheuser introduces two corresponding kinds of modality: S-modality, and Cmodality. S-modality is sensitive only to changes in substratum, whereas C-modality is sensitive only to changes in carving. The domain of S-possible worlds (the S-domain) is the set containing all and only those worlds that contain the actual carving. The domain of C-possible worlds (the C-domain) is the set containing all and only those worlds that contain the actual substratum. The D-domain contains all and only the diagonal worlds.

In accordance with RIGIDITY, the standard modality we use is a special case of S-modality (the domain of the standard possible worlds is the subset of worlds in the S-domain that are possible according to the actual world). RIGIDITY ensures that only worlds with the same carving as the actual world are possible according to standard modality (though worlds with different conventional practices are possible). Call worlds that are outside the domain of standard modality 'præterpossible worlds'. ${ }^{17}$ Any conclusions reached using præterpossible worlds are not admissible from the perspective of standard modality, as præterpossible worlds (being impossible relative to standard modality) are not relevant to the evaluation of modal claims.

Einheuser (2006, p.471) claims that conditionals like A featuring 'conventions' are ambiguous. Should they be interpreted as stipulating change along the carving or substratum dimension? She presents three disambiguations of $A$ (the claim that if our conventions had been suitably different, then a different range of necessitation claims would have been true):

Counterconventional conditional: $\mathrm{P} \rightarrow{ }_{\mathrm{cc}} \mathrm{Q}$ is true at world $w=\langle s, c\rangle$ iff $\mathrm{Q}$ at every $w^{\prime}=\left\langle s, c^{\prime}\right\rangle$ where $c^{\prime}$ differs from $c$ just enough for $P$ to be true.

Countersubstratum conditional: $P \rightarrow{ }_{c s} Q$ is true at a world $w=\langle s, c\rangle$ iff $Q$ at every $w^{\prime}=\left\langle s^{\prime}, c\right\rangle$ where $s^{\prime}$ differs from $s$ just enough for $P$ to be true.

Diagonal conditional: $\mathrm{P} \rightarrow_{\mathrm{d}} \mathrm{Q}$ is true at $w=\langle s, c\rangle$ iff $\mathrm{Q}$ at every world $w^{\prime}=\left\langle s^{\prime}, c_{s^{\prime}}\right\rangle$ where $s^{\prime}$ differs from $s$ just enough for $P$ to be true.

Depending on whether we interpret 'conventions' in A as meaning conventional practices, or carving, we can interpret $A$ as either a countersubstratum, or counterconventional conditional. As a countersubstratum conditional, $\mathrm{A}$ is false. No world $w=\left\langle s^{\prime}, c\right\rangle$ where only our conventional practices differ is a world where a different range of necessitation claims are true. Consider the necessitation claim 'necessarily, Marie Skłodowska Curie is Human if she exists'. In w' containing the actual carving and a substratum supporting different conventional practices, it is still necessary that

\footnotetext{
15 These are reformulations of Einheuser's definitions, which state that $s$ supports a feature $F$ iff there is a carving $c$ such that $\langle s, c\rangle$ has $F$, and that $c$ carves a $c$-feature $F$ iff there is a substratum such that $c$ yields $F$ when applied to $s$. I take these revisions to be beneficial.

${ }^{16}$ Note that this is not true grounding in the sense popularly discussed in contemporary metaphysics (as discussed in, for example Correia and Schnieder (2012), Fine (2012), and Trogdon (2013)), as even when the substratum and carving 'match up' in this respect, the substratum does not genuinely determine the carving. Where Einheuser uses 'grounding', one might instead use 'correspondence', or 'correlation'. Recall that there is a world for every substratum/carving combination, and it is this combination that creates diagonal worlds, not any special relationship between the substrata and the carvings. I return to this point in Section 6.

17 I use this term instead of 'impossible worlds' because such worlds are still possible relative to different modal notions.
} 
Curie is human. The modal status of Curie's humanity in $w^{\prime}$ is a product of the carving and the part of the substratum that determines that she is human (which for the neo-conventionalist has nothing to do with convention). There are only two forms of change that can affect which necessitation claims are true. The first is change in carving (which the conditional stipulates against). The second is change in the claims necessitated (for instance, if Curie were a fish), which again is stipulated against in the conditional, which singles out worlds with substrata just different enough for the conventional practices to differ (and this would represent additional differences beyond mere difference in conventional practices). Being false, countersubstratum A is no threat to the validity of S4.

As a counterconventional/diagonal conditional, $\mathrm{A}$ is true. ${ }^{18}$ In $w^{\prime}$ containing a non-actual carving and the actual substratum, it needn't be necessary that Curie is human. In all worlds $w=$ $\left\langle s, c^{\prime}\right\rangle$ where $c^{\prime} \neq c_{@}$, a different range of necessitation claims are true. Even though the claims necessitated are the same (because the substratum remains the same), a non-actual carving must produce at least one different c-feature in order to be genuinely distinct from the actual one. ${ }^{19}$ However, because standard modality is a special kind of S-modality, any such world is præterpossible relative to standard modality, and thus inadmissible when considering the validity of S4.

The falsity of countersubstratum A means that it poses no threat to S4, as it cannot play the role of $A$ in deriving the contradiction 6 in the argument above. Counterconventional $A$ is true, but is of no use in the derivation of 6 because the worlds that make counterconventional A true are Cpossible but not S-possible (making them præterpossible and irrelevant to the validity of S4, which is an axiom of modal logic applied to standard modality). This means that the possibility of a different range of necessitation claims being true is mere C-possibility, and not the possibility of standard modality. As such, counterconventional A poses no threat to S4.

The conventionalist is not committed to any countermodels to $S 4$. Only countersubstratum A would threaten the validity of $S 4$, and it is false. Counterconventional A is true, but doesn't threaten the validity of $\mathrm{S} 4$ because the worlds required for its truth are præterpossible and do not feature in the models relevant to the validity of S4. However, the truth of counterconventional A provides support for LINK because it allows there to be a sense in which $A$ is true, showing that there is a clear link between our conventions (interpreted as carvings) and the true necessitation claims. Further, that our standard notion of modality falls within S-modality shows us why we are inclined to adopt RIGIDITY (though it is worth noting that RIGIDITY is seemingly only de facto true for standard modalising, not de jure, because it just happens that no possible worlds contain non-actual carvings).

The Einheuser framework allows us to present conventionalism in a way that bears no commitment to countermodels to S4. This allows us to maintain the kind of sensible antirealism mentioned in the introduction.

\section{Applying the Einheuser Framework to Neo-Conventionalism}

The Einheuser framework is compatible with neo-conventionalist accounts of modality. The notion of a substratum is compatible with various possible worlds accounts. It could be a set of propositions, a state of affairs, or something physical, more akin to a Lewisian world. For the neoconventionalist, it is important that all of the claims necessitated that are true at a world $w$ are made true by the substratum in $w$.

More difficult is determining how one interprets the carving as taking one from a substratum to the range of necessitation claims. This cannot be done directly (that is, the carving cannot act as a function from a set of claims necessitated to a set of necessitation claims). Doing so

\footnotetext{
${ }^{18}$ Diagonal $\mathrm{A}$ is also true because all diagonal worlds with different conventional practices are counterconventional (assuming that different conventional practices cannot ground the same carving).

${ }^{19}$ Because carvings are functions, any two carvings that produce the same range of c-features (in this case true necessitation claims) from the same $s$-features are identical.
} 
would mean that, whilst the claims necessitated are not true by convention, the necessitation claims are. This is a consequence that the neo-conventionalist is eager to avoid, as it would represent a move back to traditional conventionalist reliance on truth-by-convention.

To accommodate the Humean, the carving function $c$ takes one from the substratum to a list of different 'kinds of claims', e.g. mathematical, metaphysical, etc. Any true proposition $\mathrm{P}$ of these kinds then has a corresponding true necessitation claim nec(P). Nec(P) is not true-by-convention, but true because $P$ is true and of a certain kind (this at least is the Humean line, so if it is acceptable there, then it is acceptable here).

To accommodate the deflationist, the carving function $c$ takes one from a substratum $s$ to a set of $c$-features fixing which worlds are accessible from a world $\langle s, c\rangle$. It is important to note that function $c$ does not have the worlds themselves as its output. It does not generate worlds, but rather selects a subset of independently existing worlds and bestows upon them the status of accessibility. There is a true necessitation claim nec $(\mathrm{P})$ for every proposition $\mathrm{P}$ that is true at every world that is accessible according to $c$. $\operatorname{Nec}(\mathrm{P})$ is not true by convention, but because $\mathrm{P}$ is true at every 'possible' world.

Note the potential concern that, seeing as worlds are substrata-carving pairs, there appears to be a certain amount of circularity. Worlds are individuated in terms of a substratum and a function determining which worlds are accessible to them, and in turn, these worlds are individuated in terms of other worlds that are accessible to them. Whilst it needn't matter to the deflationist which worlds are accessible from the worlds accessible from the actual world, the fact that the carvings are parts of those worlds makes it key to their identity. If world $w=\langle s, c\rangle$ accessible from the actual world were to have had carving $c^{\prime}$ instead of $c$ then it would no longer be $w$, but some other world $w^{\prime}=\left\langle s, c^{\prime}\right\rangle$. As such, each world is (partly) individuated in terms of all of the other worlds, which are in turn (partly) individuated in terms of it. One might try to present such a picture as a kind of holistic definition of the pluriverse, but one would be forgiven for thinking that the Einheuser framework is not the most natural fit for the deflationist. For now, however, I put this point aside in favour of a more pressing problem to be discussed in Section 6 . I return to this point in Section 8.

In doing this we are using conventions to select between different candidate notions of necessity, rather than making any individual necessitation claims true-by-convention. The claims necessitated we conventionally select are already true or false at a world (by virtue of whatever part of the substratum makes claims of that kind true or false) regardless of our conventions. What our conventions are doing is selecting which worlds are relevant to the evaluation of modal claims, and thus specifying one candidate notion of necessity to be of interest (the notion corresponding to the worlds our conventions deem accessible). In doing this we select a range of necessitation claims to give special significance to. However, this does not affect the threat of the contingency problem, and does not affect the Einheuser framework's potential as a response (despite Einheuser seemingly not having neo-conventionalism in mind).

\section{The Contingency Problem: Continued}

The Einheuser framework allows us to maintain S4, CONTINGENCY, LINK, and A (and on a different reading, $\neg A$ ), by distinguishing between our conventional practices and the carvings that correspond to them. By identifying an ambiguity in $A$, and exploiting the possible disambiguations, we can use the Einheuser framework to satisfy the prima facie conflicting beliefs. This allows us to present a framework in which conventionalism is true, but that is not contrary to the validity of S4. However, in the Einheuser framework LINK is supported by counterconventional A, but not by countersubstratum A. This is problematic because it is not enough that our carving should determine the range of true necessitation claims. It must also be the case that our conventional practices themselves determine our carving (and thus, indirectly, the necessitation claims). The Einheuser framework maintains a covariance claim between carvings and $c$-features at the expense 
of maintaining one between conventional practices and c-features, but the latter is the very heart of conventionalism. Any conventionalist must be able to identify that our conventional practices (and not just the carvings that form a theoretical counterpart to them) play a role in determining $c$ features. In other words, the conventionalist should want to maintain:

(LINK') There must be a clear link between our conventional practices and the true necessitation claims.

As it stands, it is the carving that is doing all of the theoretical work. There are infinitely many worlds that contain carvings that don't correspond to the conventional practices held by the inhabitants of those worlds (if there even are any inhabitants). This, combined with the fact that a world is just as likely to be non-diagonal as it is diagonal (because there are infinitely many of each), suggests that the conventional practices held at a world play no role in determining the carving in that world. Even when the substratum grounds the carving, this is just the term we apply when the two match. There is nothing in the framework that suggests any kind of actual grounding or determination. This violates LINK'. LINK' could be maintained if a conditional like B were true.

(B) If a world with suitably different conventional practices were actual, then a different range of necessitation claims would have been true. ${ }^{20}$

B would preserve LINK' because even though carvings do not correspond to conventional practices in infinitely many worlds, if any world $w$ were actual, then there is reason to claim that the conventional practices and the carving in that world would match. We want it to be the case that the conventional practices in the actual world determine the actual world's carving. ${ }^{21}$ Otherwise it is not clear that the carving has anything to do with convention, and that it is not just some philosophical trick brought into the theory to avoid a problem. B is different from $A$ because it specifies conventional practices, not just conventions, and because the antecedent stipulates that such a world be actual. When we consider a world where we hold different conventional practices (as $A$ invites us to do under one reading), the same necessitation claims hold (because of RIGIDITY). When we consider if such a world had been actual (as B invites us to do), different necessitation claims hold, because RIGIDITY stipulates that we consider hypothetical situations relative to our actual conventions. This make use of the distinction between two suppositional acts: A-supposition (roughly, supposing as actual) and C-supposition (roughly, supposing as counterfactual). ${ }^{22}$ In Asupposing that $\mathrm{P}$, we are acting as if we believe that $\mathrm{P}$ is actually the case. In doing so, we jump perspective' to that of the world(s) under consideration. A-suppositions have long been used to think about conditionals with impossible antecedents (e.g. if water had had the elemental composition $\mathrm{XYZ}$, then it would have done so necessarily). The suppositional acts associated with all of the Einheuser conditionals are C-suppositions, whereas (due to its use of the term 'actual') $B$ is clearly inviting one to engage in A-supposition. In fact, once the notion of A-supposition has been made, one might think that phrasing $B$ in terms of actuality is somewhat redundant. The genuine value of using 'actual' in B is in motivating the introduction of A-supposition, and showing that, as

\footnotetext{
${ }^{20}$ By 'actually' here I don't mean the rigidifying actuality operator signified by @, but rather actuality as a status that a world may or may not have. This is the kind of actuality that we mean when we say that it might actually have been the case that Curie was not Polish (read as metaphysical and not epistemic modality). We don't mean that she might have not been Polish in the actual world (as signified by the rigid @ operator), but that a world where she is Czech (say) might have been actual and not merely possible. There is nothing wrong with using these two notions of actuality side by side, just as there is nothing wrong with saying that every world represents itself as being actual but does not represent itself as being the actual world (signified with @). As such, I write both of 'the actual world' ( $\left.w_{@}\right)$ and of worlds being actual.

${ }^{21}$ One might think to achieve the desired result using diagonal A. However, it is built into the content of diagonal conditionals that they stipulate change both in conventional practices and in carving. As such, it only trivially maintains LINK' because, singling out only diagonal worlds, it takes us not from change in conventional practices to change in carving, but from change in conventional practice and carving to change in carving. It fails to capture the determination needed for LINK'. Furthermore, it should be the case of any standard possible world and not just the diagonal worlds that, were it actual, a different range of necessitation claims would be true.

22 cf. Chalmers (2002).
} 
we shall see, the Einheuser conditionals as presented (being instances of C-supposition) are not fit for purpose.

If some rule like B were not in effect, then we would be left with the awkward conclusion that it is mere coincidence that the actual world is diagonal, and the worrying epistemic question of how we even know that we live in a diagonal world. As such, at least in whichever world is actual, the carving should be determined by the conventional practices, establishing a clear link between the two; this is what B would do.

How does $B$ translate into the three Einheuser conditionals? The most direct reconstruction is problematic. Consider the following. Where $w^{\prime}$ is a world supporting different conventional practices and $\Phi_{w}$ is the set of necessitation claims true at $w$ :

Countersubstratum B $\left\langle w^{\prime}\right.$ is actual $\rightarrow_{c s} \Phi_{w^{\prime}} \neq \Phi_{@}>$ is true at world $w=\left\langle s_{@}, c_{@}>\right.$ iff $\Phi_{w^{\prime}} \neq \Phi_{@}$ at every $w^{\prime \prime}=\left\langle s^{\prime}, c\right\rangle$ where $s^{\prime}$ differs from $s_{@}$ just enough for $w^{\prime \prime}$ to (have different conventional practices and) be actual.

Counterconventional B $\left\langle w^{\prime}\right.$ is actual $\rightarrow_{c c} \Phi_{w^{\prime}} \neq \Phi_{@}>$ is true at world $w=\left\langle s @, c_{@}\right\rangle$ iff $\Phi_{w^{\prime}} \neq \Phi_{@}$ at every $w^{\prime \prime}=\left\langle s, c^{\prime}\right\rangle$ where $c^{\prime}$ differs from $c_{@}$ just enough for $w^{\prime \prime}$ to (have different conventional practices and) be actual.

Diagonal B $\left\langle w^{\prime}\right.$ is actual $\left.\rightarrow_{\mathrm{d}} \Phi_{w^{\prime}} \neq \Phi_{@}\right\rangle$ is true at world $w=\left\langle s_{@}, c_{@}\right\rangle$ iff $\Phi_{w^{\prime}} \neq \Phi_{@}$ at every world $w^{\prime \prime}=\left\langle s^{\prime}, c_{s^{\prime}}\right\rangle$ where $s^{\prime}$ differs from $s @$ just enough for $w^{\prime \prime}$ to (have different conventional practices and) be actual.

Countersubstratum B states that B is true iff the set of true necessitation claims is different at every world $w^{\prime \prime}$ where the substratum at $w^{\prime \prime}$ differs just enough for it to be the case that $w^{\prime \prime}$ is actual. Counterconventional $B$ states that $B$ is true iff the set of true necessitation claims is different at every world $w^{\prime \prime}$ where the carving at $w^{\prime \prime}$ differs just enough for it to be the case that $w^{\prime \prime}$ is actual. Diagonal $B$ states that $B$ is true iff the set of true necessitation claims is different at every diagonal world $w^{\prime \prime}$ where the substratum at $w^{\prime \prime}$ differs just enough for it to be the case that $w^{\prime \prime}$ is actual.

None of these conditionals are fit to play the role B was introduced for. All require that there should be some change in either the carving or substratum of a world that would make that world actual. It is not clear in any theory of possible worlds what change could take place inside a world that would give it actuality. Furthermore, each conditional has its own particular flaws.

Countersubstratum $B$ is problematic because the antecedent requires change along one dimension (change in substratum), whilst the consequent requires change along a separate dimension (change in carving). ${ }^{23}$ For the truth of the antecedent to be able to make the consequent true, the world in question must be diagonal, but the only S-possible diagonal worlds are the actual world $\left(w_{@}\right)$ and those with identical conventional practices, and in none of these is there any difference in the claims necessitated. ${ }^{24}$ Likewise, since the antecedent of B stipulates difference in conventional practices (part of the substratum) and not in carving, there can be no C-possible worlds that satisfy the antecedent, rendering counterconventional B trivially true.

The problem is that both countersubstratum and counterconventional conditionals can only function along one dimension of change. The $\mathrm{C}$-domain and the S-domain only overlap at the actual world $\left(w_{@}\right)$, and the actual world is excluded consideration by the antecedent of B. Only Diagonal conditionals can function along two dimensions of change. Diagonal B is true, but the worlds that satisfy the antecedent are præterpossible worlds and as such, do not satisfy the motivation for B because they lie outside the domain of standard modality.

\footnotetext{
${ }^{23}$ The consequent requires change in carving because without change in the claims necessitated (which is stipulated against because the antecedent allows only enough substratum change for the conventional practices to differ), the only possible source of modal change is difference in carving.

${ }^{24}$ This assumes that only one set of conventional practices can ground each carving. Whilst this is probably false, it simplifies the discussion and its falsity would not affect the result.
} 
These conditionals are unsatisfactory because they all engage in C-supposition. To accommodate $B$, the framework must be expanded to include A-suppositional equivalents of the modal notions Einheuser presents. A-countersubstratum conditionals invite us to jump perspective to worlds where we hold different conventional practices, A-counterconventional conditionals invite us to jump perspective to worlds with different carvings, but where we hold the same conventional practices, and A-diagonal conditionals invite us to jump perspective to diagonal worlds where we hold different conventional practices. As shown above, $\mathrm{C}$-suppositional conditionals are inadequate for the task. B is clearly an A-countersubstratum conditional.

The tension that made the conventionalist want to reject $A$ does not apply to $B$, because $B$ does not jeopardise the validity of S4. B does not threaten S4 for the same reason that counterconventional A does not threaten S4. S4 is a principle of standard modality, the modality based on the carving in the actual world $\left(w_{@}\right)$. The antecedent of $B$ invites us to consider a nonstandard modality, one based on the conventional practices (and thus the carving) of a non-actual world. If a different world were actual, then there would be a different group of possible worlds (worlds with the same carving). As such, the difference in necessitation claims is not relevant to the validity of $\mathrm{S4}$, because the worlds that make for this difference are præterpossible relative to $\mathrm{S} 4$.

The truth of B would allow one to maintain LINK' by demonstrating a clear connection between conventional practices and carvings. How can $B$ be (non-trivially) true?

\section{An Unsuccessful Response}

One ultimately unsuccessful attempt at a solution might be to introduce a rule like:

(DIAGONAL) The actual carving must correspond to the conventional practices in whichever world is actual.

However, this is equivalent to:

(DIAGONAL') Whichever world is actual must be a diagonal world.

violating the principle: 'if possibly $P$ then possibly actually $P$ '. ${ }^{25}$ To insist that whichever world is actual must be a diagonal world is to insist that no non-diagonal world could have been actual. If to be possible is to be possibly actual, then it must be the case that any possible world could have had the status of actuality, diagonal or not. To insist that whichever world is actual must be diagonal is to deny that there are non-diagonal possible worlds. Yet the only diagonal standard possible worlds are the actual world and those worlds that ground the actual carving, leading us to conclude that non-actual conventional practices aren't possible after all.

\section{The Proposed Solution}

As it stands, the Einheuser framework is unable to maintain LINK'. Here I suggest that the conventionalist can maintain the utility of the Einheuser framework, whilst respecting LINK', if they abandon the notion of worlds being ordered pairs of substrata and carvings. Instead, the conventionalist can identify worlds with substrata alone, and when modalising, consider propositions to be true or false at a world relative to a carving. Einheuser assents to the viability of such a position. She claims that nothing of substance hangs on such a change, because "[a]nalogues of all of the concepts [she] introduce[s ...] can be introduced for this construal of conventionalist

\footnotetext{
${ }^{25}$ Whilst I do not know of any sources that explicitly endorses such a principle, Wright suggests something like it in (1985), p.186. This principle may be considered controversial. In particular, one might be concerned that it follows from this that if $P$ is necessarily actual, then it is necessary. This however relies on conflating the two ways that $P$ could be necessarily actual. $\mathrm{P}$ could be necessarily actual in that, whatever way the world could have been, it would have actually been $\mathrm{P}$, or $\mathrm{P}$ could be necessarily actual in that the actual world (interpreted as a rigid designator) is necessarily $P$. The former is equivalent to necessarily $P$ anyway, and the latter does not allow one to infer necessarily $P$, so neither is problematic.
} 
possible worlds and the accompanying relative notion of truth at a world." ${ }^{26}$ First, one must present analogues for the three Einheuser conditionals:

Counterconventional conditional: $\mathrm{P} \rightarrow_{c \mathrm{c}} \mathrm{Q}$ is true at world $w$ relative to $c$ iff $\mathrm{Q}$ at $w$ relative to $c^{\prime}$, where $c^{\prime}$ differs from $c$ just enough for $P$ to be true relative to $c^{\prime}$.

Countersubstratum conditional: $\mathrm{P} \rightarrow{ }_{\mathrm{cs}} \mathrm{Q}$ is true at a world $w$ relative to $c$ iff $\mathrm{Q}$ at every $w^{\prime}$ relative to $c$, where $w^{\prime}$ differs from $w$ just enough for $\mathrm{P}$ to be true relative to $c$.

Diagonal conditional: $\mathrm{P} \rightarrow_{\mathrm{d}} \mathrm{Q}$ is true at $w$ relative to $c$ iff $\mathrm{Q}$ at every world $w^{\prime}$ relative to $c^{\prime}$ that is grounded by $w^{\prime}$, where $w^{\prime}$ differs from $w$ just enough for $\mathrm{P}$ to be true relative to a carving $c^{\prime}$.

We can also introduce $C$, a version of B more suitable to the revised Einheuser framework.

(C) If a substratum supporting a suitably different set of conventional practices were actual, then a different range of necessitation claims would be true.

Whilst the new conditionals are still of use in representing conventional modality, they are of little use in representing $C$. This is because, as stated earlier with $B$, it invites us to engage in Asupposition. We can take $C$ to be an A-suppositional equivalent of the countersubstratum conditional interpretation of $A$. That is, as:

(C') Under the supposition that we hold suitably different conventional practices than those in the actual world $\left(w_{@}\right)$, a different range of necessitation claims is true.

If the revised framework can maintain the truth of $C^{\prime}$ then it can preserve LINK'. One can do this by holding a principle like:

(RIGIDITY') The carving relative to which we judge worlds must correspond to the conventional practices held in whichever world is actual.

RIGIDITY' ensures that $C^{\prime}$ is true, and $C^{\prime}$ is sufficient for the conventionalist to maintain LINK'. We can see this through individual necessitation claims that would be false under the A-supposition that we hold different conventional practices. 'Necessarily, Curie is human if she exists' is true at the actual world, but if we A-suppose holding different conventional practices such that the species of biological organisms are not essential to them, then this would be false. For the purposes of this supposition, the actual world would be a world with these different conventional practices, and because of RIGIDITY' we must consider claims relative to the carving that corresponds to those conventional practices. As such, $\mathrm{C}^{\prime}$ is true because under this A-supposition it is merely contingent that Curie is human.

The reader might wonder why this move cannot be used to rescue the Einheuser framework before revising it. This would not work. In order for an A-supposition of B to be true, it would still be required that we somehow restrict A-supposition to only the diagonal worlds. Regardless of whether this would require endorsing DIAGONAL, the more pressing problem is the præterpossible status of the worlds required to make an A-supposition of B true. It should be the case of any standard possible world that if we jump perspective to that world in the way A-supposition allows then we evaluate modal claims relative to the carving grounded by that world's conventional practices. Otherwise we lack the kind of determination required to maintain LINK'. However, all standard possible worlds have the actual carving. The only worlds that would make the Asupposition of B true are præterpossible, so this approach cannot satisfy the requirement that it be true of the standard possible worlds.

Having to restrict certain suppositions to considering only diagonal worlds is a symptom of the deeper problem that in the Einheuser framework conventional practices in no way determine carvings. Even in diagonal worlds there is no reason to think that the conventional practices grounding the carving is anything more than a matter of coincidence. The proposed revision,

${ }^{26}$ Einheuser (2006), p.463 fn.7 
combined with RIGIDITY' succeeds in giving us a sense in which the conventional practices in a world do determine the carving that we consider modal claims relative to. The carving we consider a modal claim relative to is determined by the conventional practices of the world that we are supposing is actual. If we are engaging in C-supposition then that is the actual world; if we are engaged in A-supposition then that is the world we are supposing-as-actual.

When considering worlds as substrata and considering truth at worlds relative to carvings, the worlds under consideration need not be limited to only the diagonal worlds. A-supposition of $C$ only requires RIGIDITY'. RIGIDITY' is similar enough to RIGIDITY (it merely rephrases RIGIDITY in terms of the revised Einheuser framework) that it can be motivated by the same considerations that enabled Wright to present it. RIGIDITY' allows worlds to 'act' diagonal, as the worlds themselves contain no carvings that force them to be otherwise. This means that LINK' can be maintained using the standard possible worlds. That the proposed revision results in this difference demonstrates that it is a substantial revision, and not a mere notational variant of Einheuser's original framework. Furthermore, this modified Einheuser framework is also a much better fit for the deflationist. Worlds (being identified solely with substrata) are not at all individuated through carvings. This removes any risk of circularity, and provides a much more natural fit for the wider neoconventionalist picture.

\section{Guarding Against the Original Contingency Threat}

There is a risk in this approach that by maintaining RIGIDITY' and C, the position is re-exposed to the threat originally posed by $A$. We must ensure that the truth of $C$ does not jeopardise the validity of S4. Previously we had the ambiguity between the countersubstratum and counterconventional readings of the conditional to guard against this, but with no (obvious) viable counterconventional reading of $\mathrm{C}$, this option is not available. However, we can use the tools of A-supposition and Csupposition to do the same work with $C$ that Einheuser uses counterconventional and countersubstratum conditionals for.

If the $\mathrm{C}$-supposition of $\mathrm{C}$ were true then it would commit the conventionalist to a model where $\mathrm{S} 4$ is false. However, the $\mathrm{C}$-supposition of $\mathrm{C}$ is false because considered relative to the carving that corresponds to our actual conventional practices (as RIGIDITY' insists), change in conventional practices does not suffice to affect change in the range of true necessitation claims. As such, it does not commit the conventionalist to a countermodel of S4. Maintaining RIGIDITY' ensures that no such C-suppositions should arise to jeopardise the validity of S4 because the truth of any Csupposition is considered relative to our actual carving, leaving the only kind of change relevant to its evaluation as substratum difference (within the domain of worlds deemed possible by standard modality). As such, any C-supposition whose truth would be a threat to $\mathrm{S} 4$ ends up false.

The A-supposition of $\mathrm{C}$ (that is, $\mathrm{C}^{\prime}$ ) is true, but provides no countermodel to $\mathrm{S} 4$ because the antecedent of the conditional is præterpossible (in that, when combined with RIGIDITY', it invites us to 'jump perspective', thus placing us outside of the domain of standard modality). A-supposition of $\mathrm{C}$ poses no threat to $\mathrm{S} 4$ in much the same way that counterconventional $\mathrm{A}$ did not pose a threat to S4. It is made true by worlds relative to a præterpossible carving that is not relevant to axioms of modal logic for standard modality, such as S4.

\section{Conclusion}

Conventionalism, as presented here, bears no commitment to countermodels to S4. Of the conditionals considered, those that are problematic (that would require that the model be a countermodel to S4) are false, and those that the conventionalist needs to maintain are not problematic (they are made true by worlds that do not render S4 false in the model, and so do not make it a countermodel). This means that there are options available to the conventionalist that 
wants to maintain the validity of S4, leaving the ground level for arguments as to whether or not maintaining S4 is something the conventionalist ought to do. That previous responses to the contingency problem have resulted in failure to fully respect the role of convention in an account of modality is symptomatic of a common neglect to detail as to how the conventions play the role that they do. Whilst it may not be too problematic in some instances to lose track of exactly how our conventional practices work in determining modality, it is a critical problem when it leads to the conventionalist being unable to demonstrate that they do. The revised Einheuser framework at least satisfies this weaker condition by showing that the necessitation claims do covary with our conventions in the appropriate way.

\section{Bibliography}

Blackburn, Simon. "Morals and Modals". In Essays in Quasi-Realism. Oxford University Press, 1993.

Cameron, Ross. "What's Metaphysical about Metaphysical Necessity?" Philosophy and Phenomenological Research 79, no. 1 (2009): 1-16.

- - . "On the Source of Necessity." In Modality: Metaphysics, Logic and Epistemology. Oxford University Press, 2010a.

- - ". "The Grounds of Necessity." Philosophy Compass 5, no. 4 (2010b): 348-358.

Chalmers, David J. "Does Conceivability Entail Possibility?" In Conceivability and Possibility, edited by Tamar S. Gendler and John Hawthorne. Oxford University Press, 2002.

Correia, Fabrice \& Schnieder, Benjamin (2012). "Grounding: an Opinionated Introduction". In Fabrice Correia \& Benjamin Schnieder (eds.), Metaphysical Grounding: Understanding the Structure of Reality. Cambridge University Press.

Einheuser, Iris. "Counterconventional Conditionals." Philosophical Studies 127, no. 3 (2006): 459482.

Elder, Crawford L. "Conventionalism and Realism-Imitating Counterfactuals." Philosophical Quarterly no. 56 (2006): 1-15.

Fine, Kit (2012). "Guide to Ground". In Fabrice Correia \& Benjamin Schnieder (eds.), Metaphysical Grounding. Cambridge University Press 37-80.

Frege, Gottlob. Foundations of Arithmetic: A Logico-Mathematical Enquiry into the Concept of Number. Translated by Austin, J. L. Northwestern University Press, 1884/1980.

Hale, Bob. "The Source of Necessity." Noûs 36, no. s16 (2002): 299-319.

Lewy, Casimir. Meaning and Modality. Cambridge University Press, 1976.

Sidelle, Alan. "Conventionalism and the Contingency of Conventions." Noûs 43 no. 2 (2009): 224241.

Sider, Theodore. "Reductive Theories of Modality." In The Oxford Handbook of Metaphysics, 180208. Oxford University Press, 2003.

-- Writing the Book of the World. Oxford University Press, 2011.

Trogdon, Kelly (2013). "An Introduction to Grounding". In Miguel Hoeltje, Benjamin Schnieder \& Alex Steinberg (eds.), Varieties of Dependence: Ontological Dependence, Grounding, Supervenience, Response-Dependence (Basic Philosophical Concepts). Philosophia Verlag 97-122.

Van Cleve, James. "Descartes and the Destruction of the Eternal Truths." Ratio 7 no. 1 (1994): 58-62.

Wright, Crispin. Wittgenstein on the Foundations of Mathematics. Harvard University Press, 1980. 
- - . "In Defence of the Conventional Wisdom." In Exercises in Analysis, edited by lan Hacking. Cambridge University Press, 1985. 